\title{
Three-dimensional inverse design and hydrodynamic performance analysis of contra-rotating axial flow pump
}

\author{
Xiaoer Wang, Zhenshan Zhang, Meng Zhang \\ Department of Weaponry Engineering, Naval University Engineering, Wuhan 430033, China
}

\begin{abstract}
Key words: contra-rotating axial flow pump, blade design, numerical simulation, performance analysis
\end{abstract}

\begin{abstract}
In order to explore the contra-rotating axial flow pump design method and analysis its hydrodynamic performance, the inlet and outlet velocity triangle of front rotor and rear rotor was analysed, then, a high specific speed contra-rotating axial flow pump was designed using three dimensional method based on the condition of the front rotor outlet circulation equal to the inlet circulation of the rear rotor. At last, the performance of the pump is calculated by using RANS simulation. The results show that: the efficiency of the pump reaches $80.2 \%$ on the mass flow design point, namely, the average efficiency of the front rotor and rear rotor is up to $89 \%$. At the same time, the efficiency will decrease rapidly when the mass flow is greater than the design flow. The efficiency of contra-rotating axial flow pump can be maintain higher than $80 \%$ by changing the rear rotor speed when the mass flow is in the range of $0.68 Q_{d} \sim 1.07 Q_{d}$, which verifies changing rear rotor speed can effectively expand the operation range of pump's high efficiency.
\end{abstract}

\section{Introduction}

The contra-rotating axial flow pump has the advantages of large flow, high head and low speed. Therefore, it has been applied as a propulsion device in catamaran and British "stingray-1" torpedo ${ }^{[1-2]}$. the design method of pump on the current is developing, but its related performance analysis is not much.

Furukawa has designed a contra-rotating axial flow pump by experiment and tested its cavitation performance. It has been shown that the pump has A wider range of efficient ranges than traditional axial pump under the condition of the single-phase flow and the gas / water two-phase flow ${ }^{[3]}$. the lifting design method has been used to design a contra-rotating axial flow pump and its performance was qualitatively analyzed by WANG Dejun ${ }^{[4]}$. However, the above two design methods are not very good at controlling of the blade load distribution, which is likely to cause the cavitation of blade, or efficiency lower and so on.

In this paper, velocity triangles at the inlet and outlet of the contra-rotating axial flow pump are analyzed, and a contra-rotating axial flow pump was designed using the three-dimensional design theory under the condition of the outlet circulation of the front rotor equal to the inlet circulation of the rear rotor. Its hydraulic performance is quantitatively analyzed by numerical calculation method. The results show that the theoretical assumptions and design methods are accurate and effective. The efficiency of the axial flow pump will be drastically reduced When the flow exceeds the designed flow rate due to the weakening of the rear rotor load.

\section{Speed Triangles Analysis}

The speed triangles is drawing under the conditions of that the front and the rear rotor speed are equal except the direction is opposite, and that the front and rear rotor head is equal.

Assuming that the inflow velocity of the pump is axial, this means there is no circumferential component, as $V_{\text {alf }}$ shown in Figure 1 (where subscript 1 represents the inlet and subscript 2 represents the exit, subscript $\mathrm{f}$ represents Front rotor, subscript $\mathrm{r}$ represents rear rotor). the front rotor speed and the rear rotor speed is equal, but the rotation direction is opposite, then the pump peripheral speed $V_{u}=V_{u 1 f}=V_{u 2 f}=V_{u 1 r}=V_{u 2 r}$ (not consider- 
-ing the direction). The exit speed of the front rotor is equal to the inlet speed of the rear rotor. $V_{w 1 f}$ and $V_{w 2 f}$ respectively represents the relative velocity of the front rotor at the inlet or outlet. $V_{w 1 r}$ and $V_{w 2 r}$ respectively represents the relative velocity of the rear rotor at the inlet or outlet. Since the front and rear rotor has the same head, the outlet circulation of the rear rotor should theoretically be zero, which means the velocity direction of the outflow should be axial, as shown in Fig 1. The speed triangles of the contra-rotating axial flow pump is shown in Figure 1.

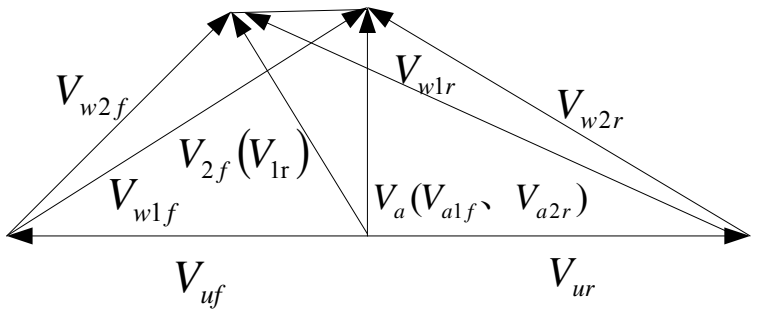

Fig. 1 Inlet and outlet velocity triangles of contra-rotating axial pump

\section{Three-Dimensional Inverse Design Theory}

Assuming that the flow in the pump is not inviscid and incompressible, at the same time, the flow before the inlet of the front rotor is irrotational. Regardless of the thickness of the blade, so that the central surface of the blade can be replaced by a vortex sheet, with the action on the water by blades replaced by the vortex on the blade, the strength of the vortex is controlled through the average circulation ${ }^{[5-6]}$ :

$$
r \bar{V}_{\theta}=\frac{B}{2 \pi} \int_{0}^{\frac{2 \pi}{B}} r V_{\theta} d \theta
$$

Where $\mathrm{B}$ represents impeller vane number, $\mathrm{r}$ represents radius and $V_{\theta}$ represents circumferential velocity. The vorticity of the flow field in the rotor can be written as follows:

$\vec{\Omega}=\nabla \times V=[\nabla \lambda \times \nabla S] \cdot \delta_{P}(S)$

Where,

$S(r, \theta, z)=\theta-f(r, z)$

$f(r, z)$ represents the wrap angle of blade, so that if the follow equation is satisfied , $\mathrm{S}$ is at the location of central surface of the blade.

$S=m \frac{2 \pi}{B}$

Where, $\mathrm{m}=1,2,3, \ldots$

$\delta_{p}(s)$ is a periodic function whose average value is one, at the same time, if $S=2 \mathrm{~m} \pi / B$, then $\delta_{p}(s) \neq 0$ otherwise $\delta_{p}(s)=0$. So that $\delta_{p}(s)$ can be expand using the Fourier series as:

$$
\delta_{p}(S)=1+\sum_{K=1}^{\infty} 2 \cos (K B S)
$$

Then, the circumferential mean rotation becomes:

$$
\vec{\Omega}=\nabla \times \bar{V}=\nabla r \bar{V}_{\theta} \times \nabla S
$$

At the beginning of the blade design, the velocity distribution in the pump is estimated according to the two-dimensional design theory under the condition of meridional shape, flow rate and rotational speed is known. Since the relative velocity $\vec{W}_{b}$ is tangent to the blade surface, The $\vec{W}_{b}$ is vertical to the normal vector of blade.

$$
\overrightarrow{W_{b}} \cdot \nabla S=0
$$

Where, 


$$
\overrightarrow{W_{b}}=\frac{W_{b}^{+}+W_{b}^{-}}{2}
$$

$W_{b}^{+}$represents the speed on the blade pressure surface, $W_{b}^{-}$represents the speed on the blade suction surface. Equation (7) is a first order hyperbolic partial differential equation whose characteristic line is the projection on the plane by the streamline on the blade. The shape of the blade is obtained by integrating along the meridional streamline ${ }^{[5-6]}$.

After obtaining the initial geometry of the blade and its velocity field, the vorticity distribution of the blade surface is obtained by using the inner flow rotation formula of the rotor, and then the three-

dimensional velocity distribution of the flow field in the rotor can be recalculated.

the three-dimensional vortex flow in the impeller can be decomposed into the circumferential mean flow and periodic pulsating flow based on reference [5-6], and the circumferential mean flow equation is derived after three-dimensional Euler equation is circumferenti -ally averaged. The periodic pulsating flow equation is established by Clebsch transformation. Then, the solution of the whole flow field is transformed into a two - dimensional Poisson partial differential equation with circumferential flow and a three - dimensional Poisson partial differential equation with circumferen -tial pulsating flow. Because the three-dimensional Poisson partial differential equation is not easy to solve, it is expanded in the circumferential direction by Fourier series, and the three-dimensional equation is transformed into an infinite number of two -dimensional equations. While, the finite terms can meet the precision requirement. After the two flow fields are solved, the finite difference method and the body coordinate system are used to solve the velocity distribution of the whole flow field in the rotor.

After obtaining the new velocity field of the pump, the shape of the blade can be obtained again by the formula (2), and then the wrap angle of the blade will be compared with the before one, if the error is out of setting error range, formula (5) will be calculated again until the error meet the requirements.

In addition, according to Kutta conditions, in order to make the outflow stable, the blade at the exit of the pressure surface and suction surface speed should be equal. According to the literature [7-8] we can see that the relative velocity increase between the blade pressure surface and the suction surface is:

$$
W_{b}^{+}-W_{b}^{-}=\frac{2 \pi}{B} \frac{\left(\nabla r \bar{V}_{\theta} \times \nabla S\right) \times \nabla S}{\nabla S \cdot \nabla S}
$$

The pressure difference between the blade pressure surface and the suction surface is obtained by simplifying the above equation using the Bernoulli equation without viscous flow.

$$
p^{+}-p^{-}=\frac{2 \pi}{B} \rho \bar{W} \cdot \nabla r \bar{V}_{\theta}
$$

Where,

$$
\bar{W}=\frac{W_{b}^{+}+W_{b}^{-}}{2}
$$

The known conditions in the design process include: the axial projection of the contra-rotating axial pump, the loading distribution along the stream line, the rotation rate, the flow rate and the number of blades.

\section{Rotor Design}

The diameter of the pump $D_{c}$ is $200 \mathrm{~mm}$, the diameter of the hub $D_{h}$ is $100 \mathrm{~mm}$, the design flow rate is $70 \mathrm{~L} / \mathrm{s}$, the front and rear rotor speed is $1225 \mathrm{r} / \mathrm{min}$, the head of the pump is $4 \mathrm{~m}$, the number of the front rotor is four and the number of the rear rotor is five . the main design parameters of the contra-rotating axial flow pump are shown in Table 1 based on the analysis of the inlet and outlet speed triangles of section 1 and the design experience of the axial flow pump. 
Table 1. Primary parameters of the contra-rotating axial pump

\begin{tabular}{ccccc}
\hline location & & hub & middle & shroud \\
\hline diameter & & 100 & 149 & 198 \\
& Stagger angle & 33.6 & 21.6 & 14.0 \\
Front rotor & solidity & 1.27 & 0.98 & 0.79 \\
& Stagger angle & 25.5 & 16.8 & 11.2 \\
\multirow{2}{*}{ Rear rotor } & solidity & 1.11 & 0.92 & 0.82 \\
& &
\end{tabular}

\subsection{Meridional Shape of The Pump}

The meridional shape of the contra-rotating axial flow pump is related to the pump's flow rate, efficiency, cavitation, and the matching of the blade. It is generally necessary to refer to the geometric dimension of the actual installation position. In this paper, the meridional shape of the contra-rotating axial flow pump is referred to the reference [9] (Fig.2)

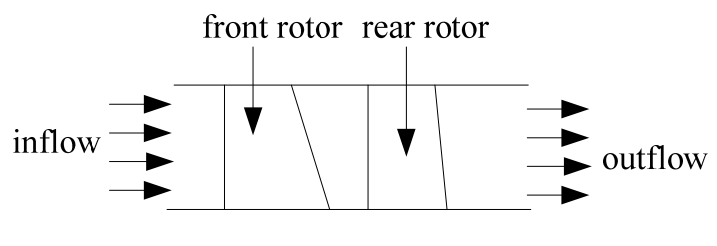

axial

Fig. 2 Meridional diagram of the contra-rotating axial pump

\subsection{Loading Distribution of Front Rotor}

The blade loading distribution of the rotor determines the efficiency and anti-cavitation performance of the rotor. Therefore, it is important to choose a reasonable loading distribution for designing a contra-rotating axial flow pump.

According to Eq. (10), it can be known that the loading of the blade is related to the partial derivative of $r \bar{V}_{\theta}$. Therefore, given the loading distribution means that the $\partial r \bar{V}_{\theta} / \partial m$ distribution along the streamline is known, which is conducive to the precise control of the loading ${ }^{[10-11]}$. The loading distribution of the blades needs to meet the following two conditions ${ }^{[12]}$ :

(1)at the Exit,

$\frac{\partial r \bar{V}_{\theta}}{\partial m}=0$

This is because under the Kutta condition, $p^{+}=p^{-}$should be satisfied at the blade trailing edge, therefore,

$$
\bar{W} \cdot \nabla r \bar{V}_{\theta}=0
$$

So that,

$$
\frac{\partial r \bar{V}_{\theta}}{\partial m}=0
$$

Because of $\bar{W} \neq 0$ Under normal circumstances.

(2)The blade leading edge to the trailing edge of the loading integral value should equal to the circulation at the trailing edge, namely:

$$
\int_{0}^{1} \frac{\partial r \bar{V}_{\theta}}{\partial m} d m=V_{\theta_{2}} r_{2}
$$

Where, $m=0$ represents blade leading edge $m=1$ represents blade trailing edge, the subscript 2 represents the outlet, and $V_{\theta 2} r_{2}$ is the speed moment at the outlet.

Reference [10-12] shows that the maximum efficiency of the axial flow pump loading distribution is fore-loaded type, that means the blade loading from the leading edge position began to increase rapidly in the $20 \%$ chord length to reach the maximum, and then slowly decline, at last, become to 0 at the trailing edge. This form of loading distribution can effectively reduce the flow separation and improve efficiency. But this loading distribution also makes the blade suction surface pressure drop 
area larger, which is not conducive to the anti-cavitation performance of the blade. The post-loaded type is better for blade anti - cavitation performance, but the efficiency is lower than the fore-loaded type. This paper takes into account the efficiency and cavitation of the axial flow pump, the maximum loading is used at $20 \%$ chord length, and then slightly decline until $60 \%$ chord length for shroud. While the maximum loading of the hub is used at $60 \%$ chord length. The specific loading distribution of the front rotor is shown in Fig. 3, and the other radial section loading distribution are obtained by linear interpolation.

The rear rotor is operated in the outflow of the front rotor and is opposite to the direction of rotation of the front rotor, so that the inlet circulation of the rear rotor is negative and equal to the front rotor exit circulation according to section 1 . An appropriate attack angle can be chosen in the design process, which means it should take a positive value at the leading edge in Figure 4. In this paper, the values of the hub and shroud at the leading edge are 0.4 and 0.5 , respectively, after a slight rise, then decreases rapidly at $60 \%$ chord length. The specific loading distribution is shown in Fig4. The loading distribution at the other radius is obtained by linear interpolation.

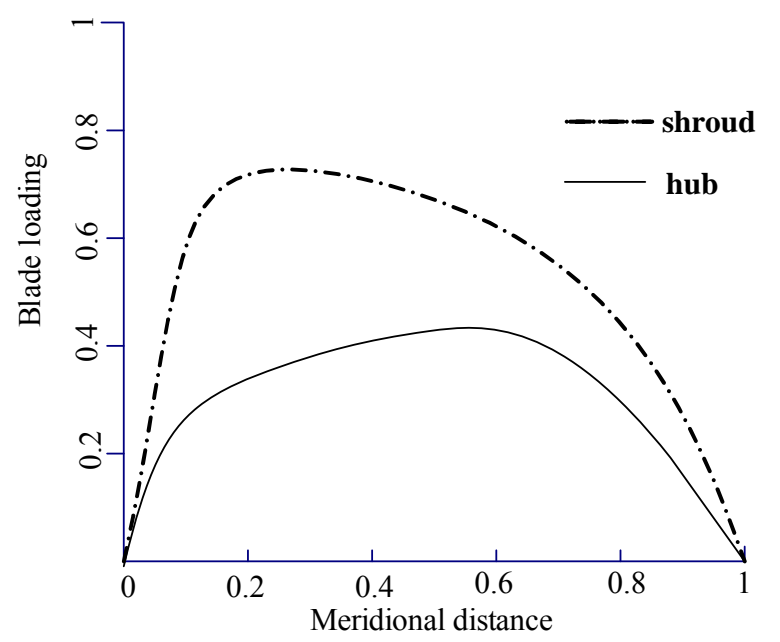

Fig. 3 Blade loading distribution of the front rotor

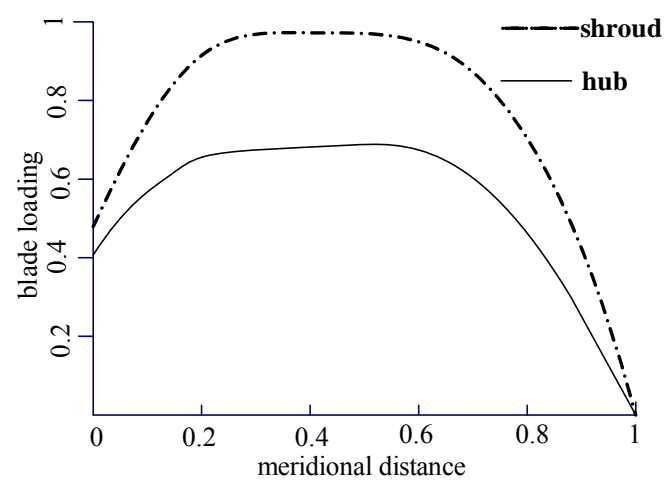

Fig. 4 Blade loading distribution of the rear rotor

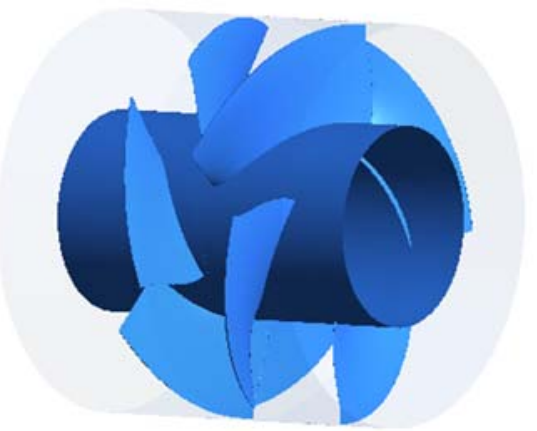

Fig. 5 Geometry of the contra-rotating axial pump 


\subsection{Blade Thickening}

After the no-thickness blade is obtained, the blade is thickened based on NACA series of airfoil thickness distribution rules. The maximum thickness of the rotor hub is $6 \mathrm{~mm}$ and the maximum thickness of the blade tip is $4 \mathrm{~mm}$. In the reference [13], in order to suppress the secondary flow of the blade, the maximum camber of the blade at the hub is placed at $60 \%$ chord length, the maximum camber at the shroud is placed at $40 \%$ chord length, and the remaining camber position is obtained by interpolation. The final geometry of the contra-rotating axial flow pump is shown in Fig 5 .

\section{Performance Analysis of Contra-Rotating Axial Flow Pump}

After obtaining the geometry of the contra-rotating axial flow pump, the central node control and the finite volume method is used to solve the RANS equation. And the SST turbulence model is used to make the equation closure.

the grid of rotor is divided by Turbo-Grid. The topology model is chosen to be J-type, the blade tip gap is $1 \mathrm{~mm}$, the number of front rotor single channel grids is 75,000 , the number of rear rotor single channel grids is 60,000 . front and rear rotor speed were set to $1225 \mathrm{r} / \mathrm{min},-1225 \mathrm{r} / \mathrm{min}$. The hub of front and rear rotor are set to relatively static wall boundary, and the shroud of front and rear rotor are set to absolute Static wall boundary ${ }^{[14]}$.

The calculation results are shown in Fig 7. It can be seen from the figure that the efficiency of the pump is high in the vicinity of the design flow rate. the efficiency of the pump is kept above $74 \%$ in the range of $55 \mathrm{~L} / \mathrm{s} \sim 75 \mathrm{~L} / \mathrm{s}$. According to the total efficiency of the pump $\eta_{p}=\eta_{f} \cdot \eta_{r}$, The average efficiency of single rotor is more than $86 \%$. At the same time, when the flow rate of the pump exceeds the designed flow rate, the efficiency of the pump will be rapidly reduced, which has been confirmed by the experimental results in reference[5]. The pump head is 2.91 meters at the design flow, lower than the design head, which shows that in the design of contra-rotating axial flow pump, according to the front and rear rotor speed consistent, head to the same criteria to design the blade, it is difficult to achieve the design head in the design flow rate. When the flow rate is $67 \mathrm{~L} / \mathrm{s}$, the maximum efficiency of the pump reaches $80.9 \%$, which means the average efficiency of single rotor close to $90 \%$.

Compared with the traditional axial flow pump, the contra-rotating axial flow pump not only can reduce the load of the blade, improve the cavitation performance of the blade, but also can ensure the efficiency of the whole axial pump by adjusting the rotational speed of the rotor. According to the literature [13], it is better to the pump's anti-cavitation performance if the front rotor speed is higher than the rear rotor. In the non-design flow conditions, the mismatch problem of the front rotor outflow angle and the rear rotor inflow angle can be overcame by changing the rotor speed, thereby enhancing the overall efficiency of the pump keep higher. Figure 8 is drowned under the condition of front rotor speed unchanged, only to change the speed of the rear rotor. the pump efficiency has a significant improvement compared to the front and rear impeller speed consistent situation in the range of $Q>40 \mathrm{~L} / \mathrm{s}$. In the range of $0.68 Q_{d} \sim 1.07 Q_{d}$, the efficiency of the pump can be maintained up $80 \%$, which means the average efficiency of a single rotor is more than $89 \%$. In the actual application process, it will be better through intelligently adjusting the speed of the front or rear rotor to ensure greater efficiency in the flow rate range, which fully proves the contra-rotating axial flow pump has a superiority in the adjusting of head and efficiency. 


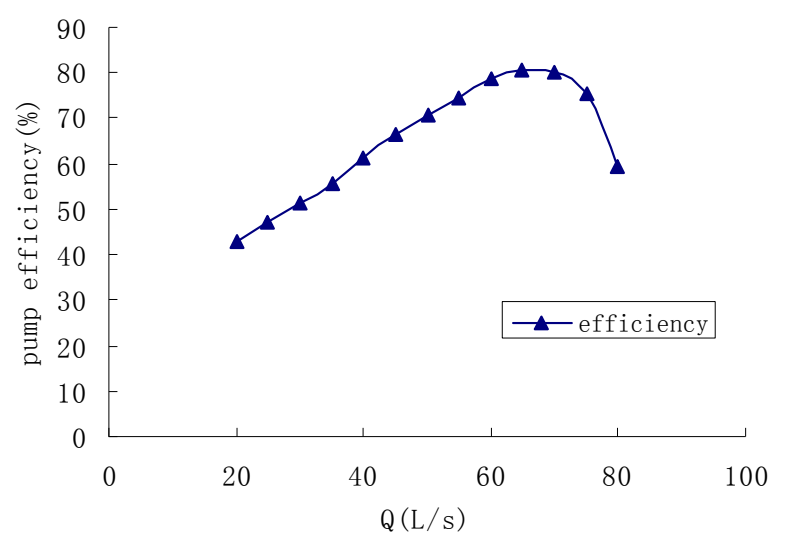

Fig. 6 Q-efficiency curve of contra-rotating axial pump

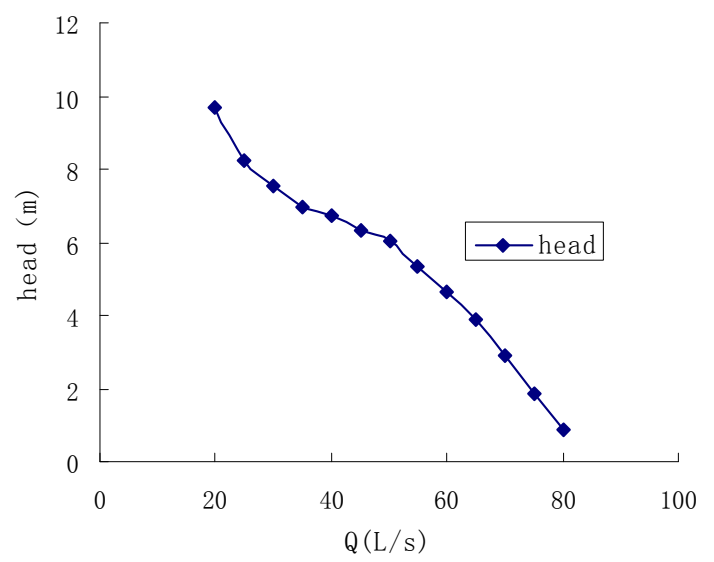

Fig. 7 Q-head curve of contra-rotating axial pump

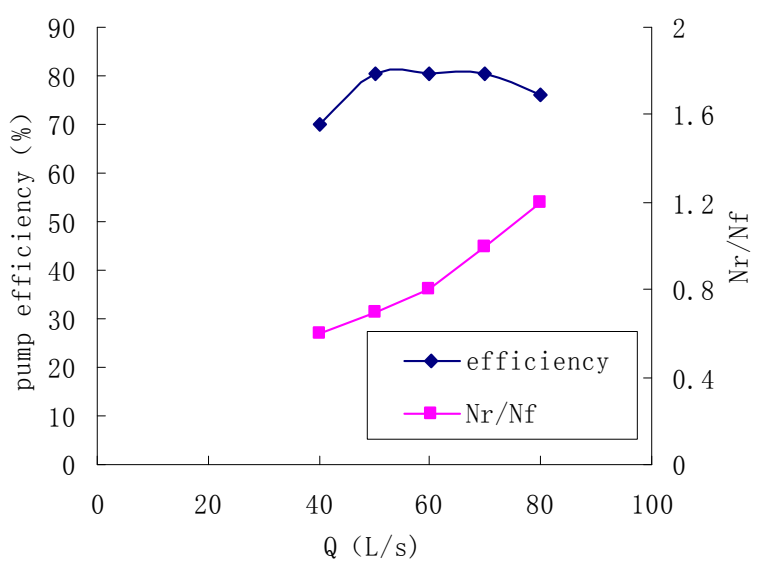

Fig. 8 Pump characteristics curve by changing $\mathrm{Nr} / \mathrm{Nf}$

\section{Conclusion}

(1) A contra-rotating axial flow pump is designed by three-dimensional inverse method which is feasible and faster. Blade loading distribution is used to control the geometry of the blade, thus controlling the efficiency and anti-cavitation performance of the blade, which is direct and effective. The positive incident angle of the rear rotor ensures the efficiency of the pump under the design flow rate, but the head cannot meet the designer requirements, and further analysis is needed.

(2) The interaction between the front and rear rotor of contra-rotating axial flow pump is very intense. The pump has a wide range of high efficiency when the flow rate is less than the designed flow rate, but due to the weakening of the rear rotor load, The efficiency of the pump will be 
drastically reduced when the flow rate exceeds the designed flow rate. The design of the rear rotor is critical for contra-rotating axial flow pump to achieving high efficiency.

(3) The structure of the contra-rotating axial flow pump is better in reducing the pump speed. by adjusting the front rotor or rear rotor speed can effectively improve the pump performance, which makes the pump have a high hydraulic efficiency in a larger flow range, it is also a significant advantage over traditional axial flow pump.

\section{Reference}

[1]. A. Furukawa, Y. Cao, K. Okuma, S. Watanabe, 2000, "Experimental Study of Pump Characteristics of Contra-Rotating Axial Flow Pump," Proc. 2nd International Symposium on Fluid Machinery and Fluid Engineering, Beijing, China, pp. 245-252.

[2]. Wang Lan, The history of torpedo development in the world and its landmark study [J]. Underwater weapons, 2015, 2:1-9.

[3]. A.Furukawa, T.Shigemitsu, S.Watanabe. Perfor -mance test and flow measurement of contra rotating axial flow pump. Journal of Thermal Science 2006,Vol.16, No.1 7-13.

[4]. Wang Dejun, Zhou Huizhong, Huang Zhiyong.Research on hydraulic performanc of contrarotating axial flow pump [J].Nuclear Power Engineering,2004,25 (1):59-62.

[5]. Luo Xingqi. Full three-dimensional inverse and optimum design of Francis runner [D].Beijing: Tsinghua University, 1995:25-84.

[6]. Peng Guoyi. Three-dimensional rotational flow inverse computation and optimum design of Kaplan runner [D]. Beijing: Tsinghua University,1996:56-70.

[7]. Zangeneh.M. A compressible three dimensional blade design method for radial and mixed flow turbomachinery blades [J].Journal of Numerical Methods in Fluids,1991,13:599-624.

[8]. Tan C S, Hawthrone W R, Mccune J E, et al. Theory of blade design for large deflections: part 2 annular cascades [J]. ASME Journal of Engineering for Gas Turbines and Power,1984, 106 (2):354-365.

[9]. Y. Cao, A. Furukawa, Quasi-3D inviscid flow analysis of contra-rotating axial flow pump [J].Memoirs of the faculty of engineering, 2000, 60 (4):143-154.

[10]. Duccio Bonaiuti, Mehrdad Zangeneh. Parametric Design of a Waterjet Pump by Means of Inverse Design, CFD Calculations and Experimental Analyses [J].ASME Journal of Fluids Engineering, 2010, 132 (1):1-15.

[11]. Duccio Bonaiuti, Mehrdad Zangeneh. On the coupling of inverse design and optimization techniques for the multi-objective, multipoint design of turbomachinery blades [J].ASME Journal of Turbomachinery, 2009, 131 (2): 1014-1030.

[12]. Zangeneh.M, Goto.A, Takemura T. Suppression of secondary flows in a mixed-flow pump impeller by application of three-dimensional inverse design method: part1: design and numeri- cal validation [J]. ASME Journal of Turbo- -machinery, 1996, 118: 536-543.

[13]. L. Cao, S. Watanabe, S. Momosaki, T. Imanishi and A. Furukawa, 2012 "Low Speed Design of Rear Rotor inContra-Rotating Axial Flow Pump," Proc. 6th International Symposium on Fluid Machinery and Fluid Engineering, Jeju Korea, REF-1150

[14]. Jin Shuanbao, Wang Yongsheng, Ding Jiangming. Three-dimensional design and numerical experiment of mixed-flow waterjet with CFD [J]. Journal of Harbin Engineering University, 2012, 33 (10):1223-1227. 